\title{
Abdominal Myomectomy for Huge Uterine Myomas with Intra-arterial Balloon Occlusion: Approach to Reduce Blood Loss
}

\author{
Satoru Takeda, MD, PhD ${ }^{1}$ Tsuyoshi Ota, MD, PhD ${ }^{1}$ Hiroshi Kaneda, MD, $\mathrm{PhD}^{1}$ \\ Yasuhisa Terao, MD, $\mathrm{PhD}^{1}$ Ryohei Kuwatsuru, MD, $\mathrm{PhD}^{2}$
} 1 Department of Obstetrics and Gynecology, Faculty of Medicine,
Juntendo University, Tokyo, Japan
2 Department of Radiology, Faculty of Medicine, Juntendo University,
Tokyo, Japan

\begin{abstract}
Address for correspondence Satoru Takeda, MD, PhD, Department of Obstetrics and Gynecology, Faculty of Medicine, Juntendo University, 2-1-1, Hongo, Bunkyo-ku, Tokyo, Japan 113-8421

(e-mail: stakeda@juntendo.ac.jp).
\end{abstract}

Surg J 2020;6(suppl S1):S11-S21.

\begin{abstract}
Keywords

- abdominal myomectomy

- hemorrhage

- huge myoma

- intra-arterial balloon occlusion

- uterine cervical myoma

Abdominal myomectomy for a huge myomas, especially uterine cervical myoma, is difficult because of risks, such as intraoperative bleeding or injury to adjacent organs. Therefore, understanding of the positional relationships among a huge myoma, especially cervical or intraligamental myoma, and the vascular plexuses in the right and left cardinal ligaments is important for prevention of massive bleeding during myomectomy. While sufficiently performing preoperative assessment with pelvic examination, ultrasonography, magnetic resonance imaging (MRI), etc., surgeons should always keep in mind how they can reduce the blood loss volume, while safely and surely performing resections. For a cervical myoma of the uterus and giant uterine leiomyoma that leave no intrapelvic space and prevent palpation and identification of the uterine arteries and the internal iliac arteries, surgery can be performed safely by preoperatively placing balloon catheters in the internal iliac arteries. Hemostaic strategies for myomectomy and tips of subsequent pregnancy following myomectomy are also described.
\end{abstract}

\section{Preoperative Evaluation}

Pelvic examination is performed to determine the location of the vaginal portion of the cervix, deviation state (whether the deviated vaginal portion is located anterior or posterior to the cervical myoma), the portion of the posterior vaginal wall where myoma is palpable, etc. Transabdominal and transvaginal ultrasonography should be always performed to determine the size, location, number, etc., of myomas and the location of the uterine corpus, uterine cavity, cervix, and cervical canal. Whether deep vein thrombosis has been caused by compression due to a giant myoma should be confirmed to see the blood flows in the bilateral femoral veins by ultrasonography. Magnetic resonance imaging (MRI) should be performed to preoperatively determine the portion of the uterine cervix from which a myoma arises, the length of the cervical canal, locations of the cervical canal, uterine body, ovaries and the cardinal ligaments including in uterine arteries and uterine venous plexus, etc. (-Fig. 1). It is important to know whether myoma is located in the anterior wall or in the posterior wall, especially in a case with a huge uterine cervical myoma, because the uterine incision must be decided preoperatively for myomectomy. And MRI should be also performed to differentiate myoma from uterine sarcoma.

Intravenous pyelography or contrast-enhanced computed tomography (CT) should be performed to understand the severity of hydronephrosis, the stenotic site of the ureter and the degree of deviation of the ureters and
DOI https://doi.org/ 10.1055/s-0039-1693041. ISSN 2378-5128.
Copyright $(2020$ by Thieme Medical Publishers, Inc., 333 Seventh Avenue, New York, NY 10001, USA. Tel: $+1(212)$ 760-0888.
License terms

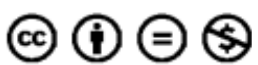




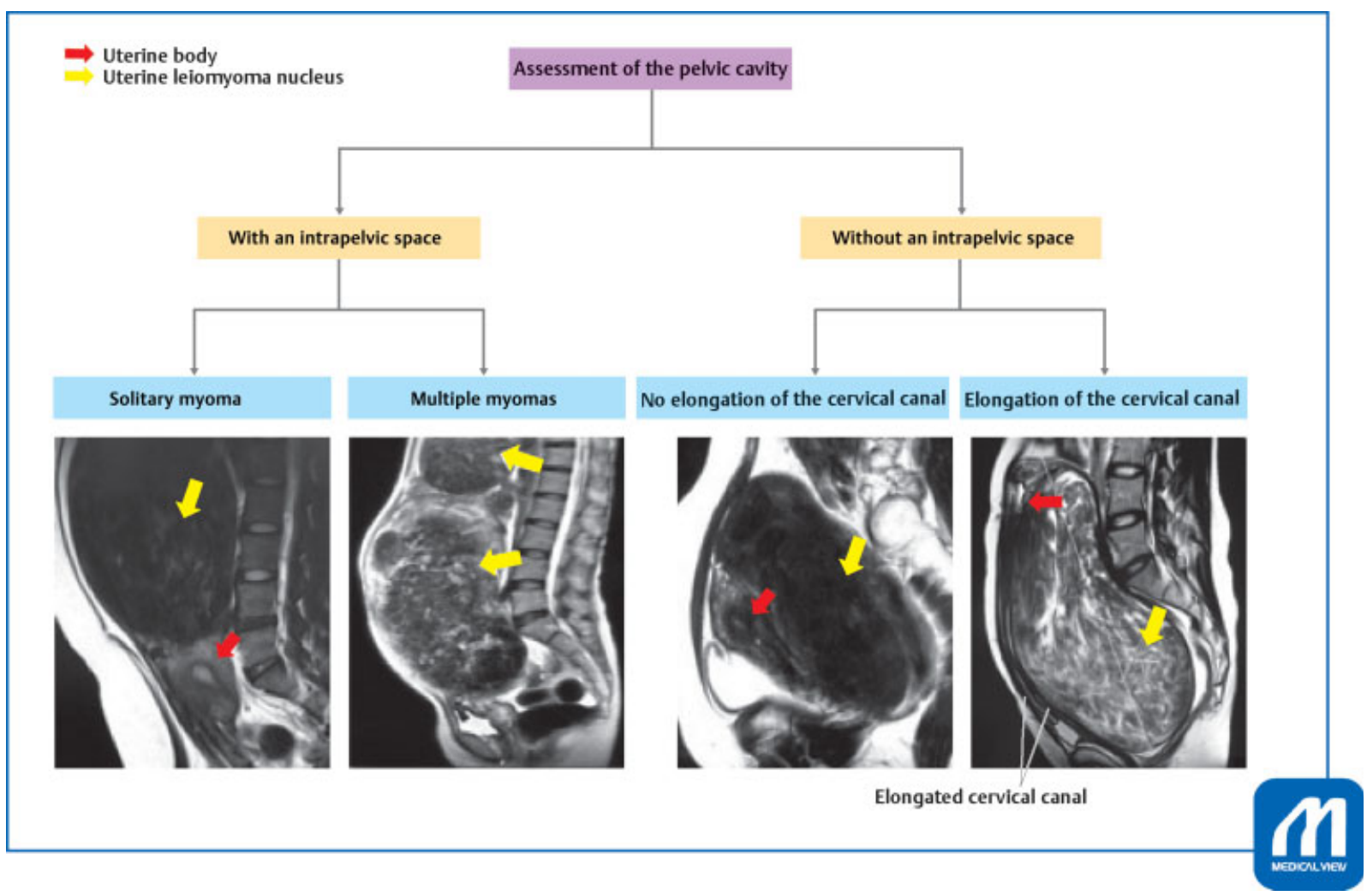

Fig. 1 Preoperative assessment by diagnostic imaging: assessment is performed to determine the presence or absence of a space between the uterus/myoma nucleus and the pelvic wall, the locations of the uterine body, uterine cervix, and cervical canal, etc. Based on the assessment results, the portion of the uterus from which the myoma nucleus arises can be determined. (Reproduced with permission from Takeda $S$. Abdominal myomectomy for huge myomas. In: Hiramatsu Y, Konishi I, Sakuragi N, Takeda S, eds. Mastering the Essential Surgical Procedures OGS Now, No.24 [Japanese]. Tokyo: Medical View; 2015: 68-87. Copyright @ Medical View).

urinary bladder. In addition to preoperative conventional examinations, thrombotic markers, such as D-dimer and soluble fibrin monomer complex, are assessed. When venous thromboembolism is suspected, a definitive diagnosis is made by ultrasonography of the lower extremities or contrast-enhanced CT.

\section{Preoperative Preparation}

The preoperative use of gonadotropin releasing hormone (GnRH) agonists contributes to reduction in not only myoma size and skin incisions but also the volume of blood loss. ${ }^{1}$ Although a cervical myoma is often less responsive to the cytoreductive effect of GnRH agonists than a myoma of the uterine body, these agents are useful because they allow correction of anemia and autologous blood collections. In surgery performed, while on heparin for antithrombotic therapy, the volume of blood loss increases even when occlusion catheters are preoperatively placed in the internal iliac arteries. Thus, autologous blood transfusion could be planned and collected, and intraoperative blood salvage system (Cell Saver) could be prepared in an operating room.

Preoperative ureteral stent placement facilitates identification of the courses of the ureters and contributes to prevention of ureteral injury, if possible or in case with hydronephrosis. When fresh thrombi are detected, surgery is postponed until their complete resolution in response to heparin therapy or an inferior vena cava (IVC) filter is placed to trap free thrombi and to prevent pulmonary thromboembolism under heparin therapy.

Before starting the operation, another pelvic examination is performed under anesthesia to confirm the location of the vaginal portion of the cervix, as well as the site, size, mobility, etc., of a mass. Placement of a balloon catheter for tubal patency test or other devices in the cervical canal facilitates identification of the cervical canal and prevents injury to the canal during surgery. If a catheter cannot easily be inserted, it should be inserted after myomectomy has progressed to a certain point. Such catheters are important for prevention of injury to the cervical canal and stenosis during suturing. If a large myoma is attached to the cervical canal, the mucosa of the cervical canal may be become detached after myomectomy.

For a giant myoma nucleus and cervical myoma buried in the small pelvis, myomectomy is performed with occlusion catheters preoperatively placed in the internal iliac arteries in order to reduce bleeding and thereby possibly avoid homologous blood transfusion. In general, requests for catheterization are made to the radiology department, and 
balloon occlusion catheters are inserted under fluoroscopic guidance immediately before surgery.

\section{Tips and Warnings Based on Anatomical Features}

Understanding of the positional relationships among a

huge myoma, especially cervical or intra-ligamental myoma and the vascular plexuses in the right and left cardinal ligaments is important for prevention of massive bleeding during myomectomy. The vascular plexuses in the cardinal ligaments are extended up and down and right and left because of the growth of myoma; consequently, they are distributed to cover the myoma surface. Thus, more blood vessels than normal are located and dilated on the myoma surface, which can be a source of massive bleeding in the event of an injury. For myomectomy of cervical myoma, it is important to know which cervical wall (i.e., anterior, posterior, or lateral wall) a myoma arises from. In a patient with a myoma arising from the lateral wall, knowing that the myoma arises from a section posterior or anterior to the cardinal ligament is important (-Fig. 2). A larger cervical myoma makes identification of its origin difficult. It should preoperatively be identified by determining the location of the vagina by pelvic examination, examining MRI, etc. The origin of cervical myoma is the most important information for developing surgical strategies regarding myoma incision sites, approaches, detachment directions, etc., for myomectomy. In a patient with a large cervical myoma, the cervical canal deviates, and its lumen cannot be located. There is a risk of severing the cervical canal during myomectomy. In a patient with a cervical myoma arising from the anterior wall, the ureters and urinary bladder may be elevated.

Both a very large cervical myoma and a myoma growing in the broad ligament are always attached to a thin muscle layer on their surfaces. In principle, this muscle layer is incised to enucleate the myoma nucleus.

The blood vessels in the muscle layer branch from the ascending and descending branches of the uterine arteries and run in a circular pattern. Based on this anatomical feature of these vessels, the least vascularized sections are the median parts of the anterior and posterior uterine walls and uterine fundus. This median part of the anterior uterine wall is also the site of uterine rupture during pregnancy in women with previous classical cesarean section. Making an incision at these sites should be avoided whenever possible. In principle, a transverse incision and vertical suture are used for myoma of the uterine body and cervical myoma, and a vertical incision and transverse suture for myoma of the uterine fundus.

A cervical myoma buried in the pelvic cavity is difficult to lift. If the uterus is pulled with excessive force, the vascular plexuses in the cardinal ligaments are at high risk of being severed. Consequently, massive bleeding can occur, requiring damage control surgery or transcatheter arterial embolization (TAE). In some cases, massive bleeding necessitates massive blood transfusion and can even lead to intraoperative death.

The ureters are located close to a cervical myoma. Their courses should always be determined up to the tunnels of the cardinal ligament before myomectomy. In a patient with a myoma arising from the anterior cervical wall, the urinary bladder is elevated and deviated, and the cardinal ligament is also dorsally deviated. Thus, the courses of the ureters should be traced anteriorly beyond the crossing sites in the cardinal ligament up to the urinary bladder. Preoperative ureteral stent placement facilitates identification of the ureters and contributes to prevention of injury to these structures. When the infundibulopelvic ligament is traced superiorly, the ureter, which runs close to the ligament, can be identified easily. In a patient with a large myoma, if the ureters, the blood vessels of the cardinal ligament and the infundibulopelvic ligaments are identified, vessel tape should be passed around them as identifying marks.

\section{Approaches to Reduce Intraoperative Blood Loss}

\section{Local Injection of Vasopressin}

Vasopressin (Pitressin;20 units $/ 1 \mathrm{~mL}$ ) is diluted with $100 \mathrm{~mL}$ (0.2 units $/ \mathrm{mL}$ ) of physiological saline for use. When 4 units $(20 \mathrm{~mL})$ of Pitressin diluted 100-fold with physiological saline are locally injected into the myometrium, the uterine surface becomes pale immediately after injection and becomes red again approximately 20 minutes later. The effect of vasopressin lasts approximately 20 to 30 minutes.

Serious adverse effects of vasopressin include hypotension, cardiac arrest, and pulmonary edema. Because adverse effects caused by injecting a diluted vasopressin solution at a high concentration of 0.5 to 0.6 unit/mL, or even higher, it is essential to adhere to the dilution rate specifications.

\section{Placing a Tourniquet around the Lower Uterine Segment}

A stoma is created in the broad ligament around the uterine lower segment. A rubber tube is inserted through the stoma and tightly tied at the level of the internal os. Although this is the simplest and most commonly used method, it is not indicated for patients in whom it is difficult to obtain a view of the uterine cervix because of a cervical myoma of the uterus or giant uterine leiomyoma or those with a highly occluded Douglas pouch. Because veins are also compressed simultaneously, this method may cause congestion in the uterus and increase bleeding in a case with a huge myoma.

\section{Clamping of the Uterine and Ovarian Arteries}

Once the retroperitoneal space is exposed in cases with an intrapelvic space, only the main trunks of the uterine arteries or internal iliac arteries are separated and clamped with bulldog clamps or other devices. As there is no venous 


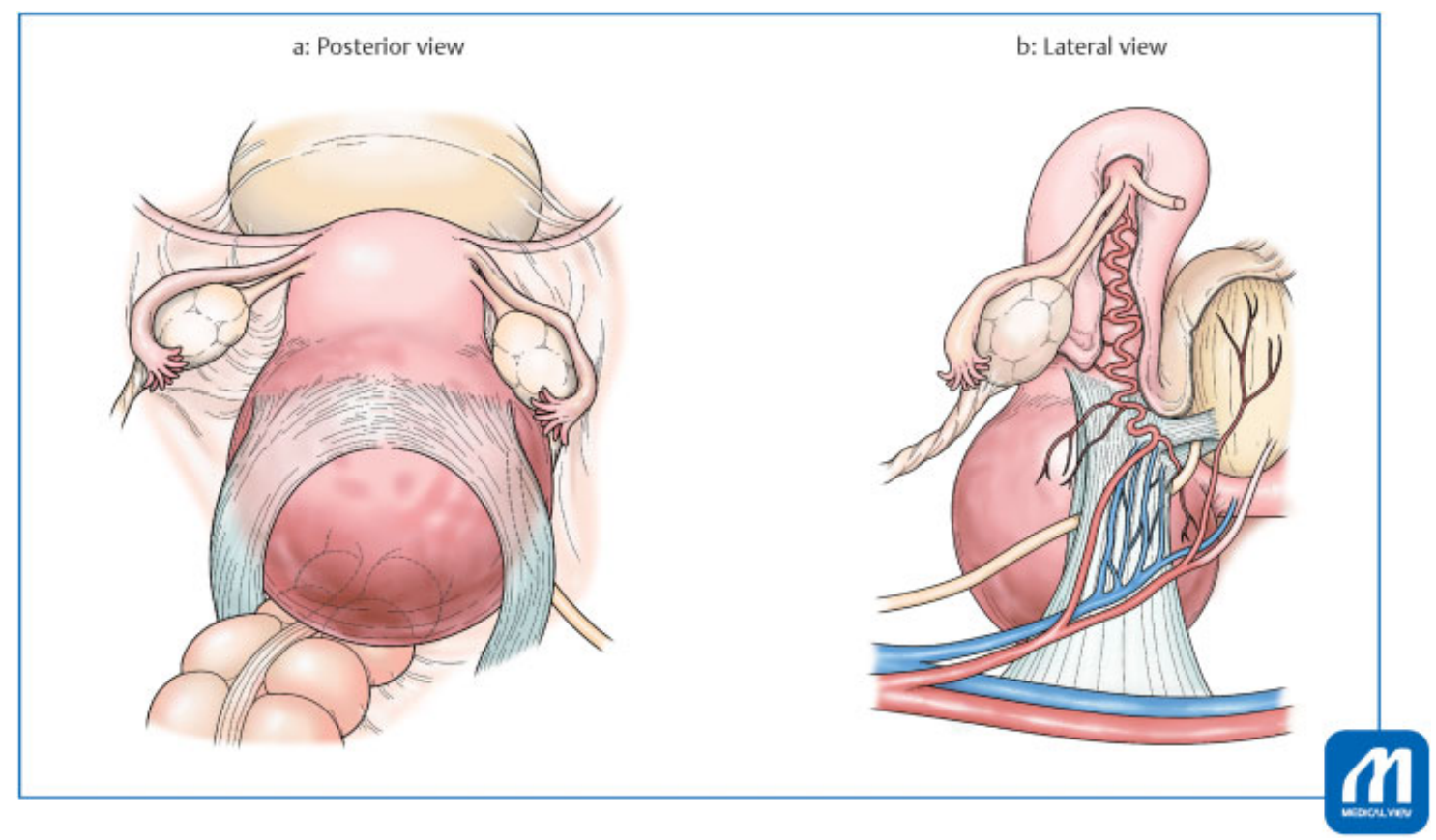

Fig. 2 Cervical myoma. (Reproduced with permission from Takeda S. Abdominal myomectomy for huge myomas. In: Hiramatsu Y, Konishi I, Sakuragi N, Takeda S, eds. Mastering the Essential Surgical Procedures OGS Now, No.24 (Japanese). Tokyo: Medical View; 2015: 68-87. Copyright $\odot$ Medical View.)

compression, congestion in the uterus can be reduced. In a patient with a giant myoma, because of abundant blood flow from the ovarian arteries, the ovarian arteries and veins are separated and vessel tape is passed around them. The avascularization achieved by clamping these arteries with blood vessel clamps or VASCU-STAT ${ }^{\circledR}$, etc allows further reduction in blood flow to the uterus.

\section{Internal Iliac Artery Balloon Occlusion (IIABOC)}

Indication of this IIABOC is myomectomy for a huge uterine cervical myoma and giant uterine leiomyomas that leave no intrapelvic space and prevent palpation and identification of the uterine arteries and the internal iliac arteries. ${ }^{1-3}$

Balloon catheters are inserted from each side of the groin and placed in the contralateral internal iliac arteries by the Seldinger technique. Then, the balloons are inflated to interrupt the blood flow to the uterine arteries. The balloons are placed distal to the superior gluteal arteries ( - Fig. 3). How much the balloons need to be inflated to interrupt blood flow should be determined. When balloon catheters are placed in the internal iliac arteries, heparin is not needed to prevent arterial thrombosis. When they are placed in the common iliac arteries or the abdominal aorta, heparin is intravenously injected at 3,000 to 5,000 units before dilatation of the balloons.

The maximal allowable duration of blood flow interruption is 60 minutes. When blood flow must be interrupted for a longer period, the interruption should be released once. During surgery, percutaneous arterial blood oxygen saturation meters should be attached to the right and left first toes. Because this procedure is also applicable to obstetrical patients with placenta previa accrete or placenta percreta, close cooperation with the radiology department should be established. ${ }^{4-7}$ Pre- operative transcatheter arterial embolization (TAE) is contraindicated in the presence of adjacent organ injury, for which healing is impaired when blood flow is decreased.

\section{Indications and Features of Arterial Balloon Occlusion in Myomectomy}

However large the uterus is, if there is a space between it and the pelvic wall, the uterus can be elevated or the tourniquet method can be applied. For such cases, arterial balloon occlusion is not indicated. Interventional radiology (IVR) is not indicated for patients in whom the ascending branches and main trunks of the uterine arteries and internal iliac arteries can be clamped with blood vessel clamps after exposure of the pelvic retroperitoneum or to whom the tourniquet method is applicable (-Fig. 1, - Table 1). The reason for this is that arterial puncture may cause complications, such as hematoma, false aneurysm, arterial thrombosis, balloon rupture, venous thrombosis due to compression of the puncture site and unnecessary radiation exposure.

In contrast, even for a not very large myoma that arises from the cervix or a huge intraligament myoma and is buried in the pelvis, arterial balloon occlusion is indicated. If the uterus is pulled with excessive force to secure a good view of the surgical field, the arterial and venous plexuses in the cardinal ligaments may be severed, which will result in massive bleeding. If myomectomy is performed with only local injection of vasopressin without interruption of blood flow or with only Cell Saver, bleeding may unexpectedly progress to massive bleeding.

During myomectomy of a cervical myoma, bleeding may increase despite both the ovarian and the uterine arteries being interrupted. This suggests that blood flow from the 


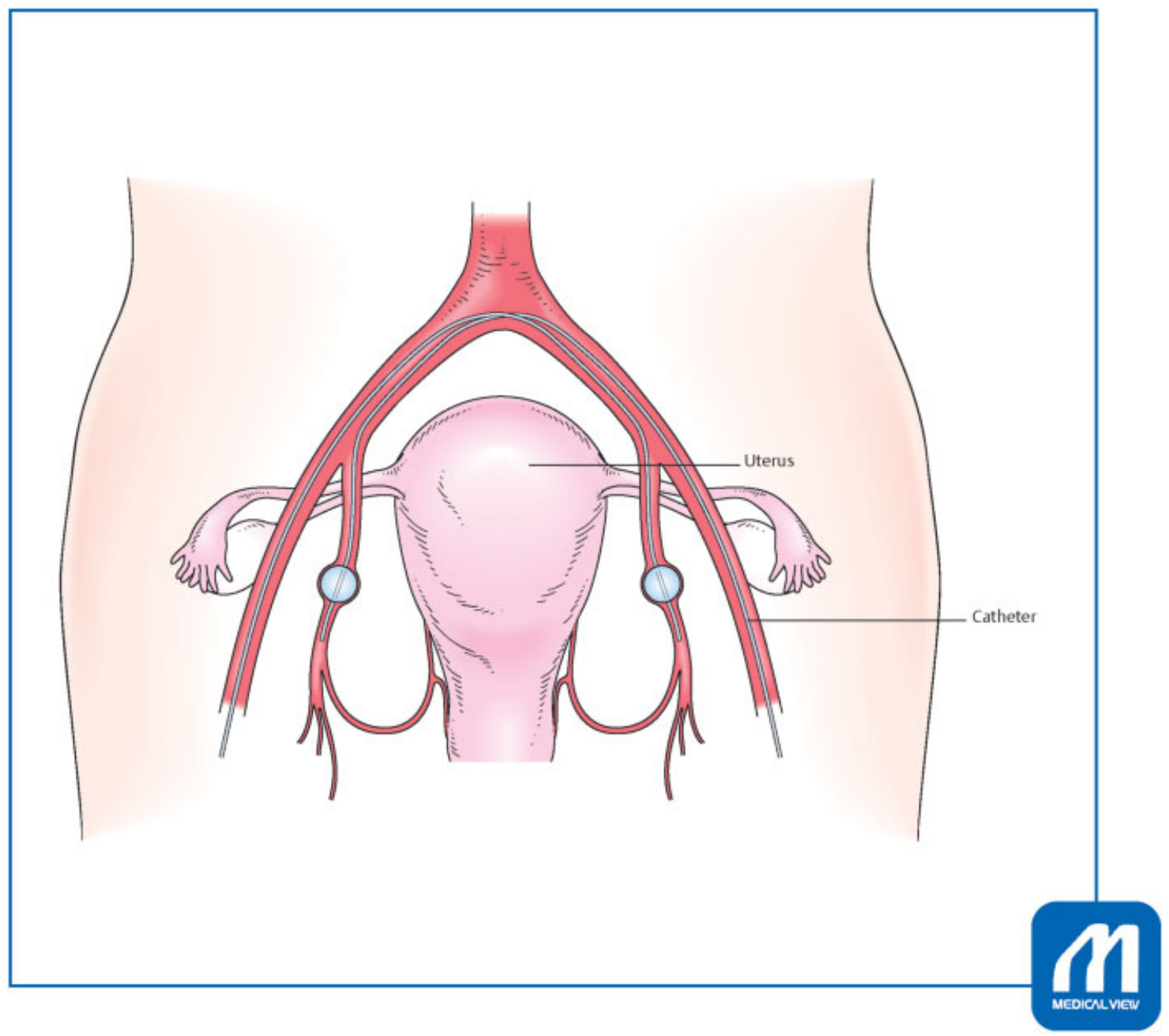

Fig. 3 Temporary balloon occlusion of the internal iliac artery: balloon catheters are inserted from each side of the groin and placed in the contralateral internal iliac arteries employing the Seldinger technique. Then, the balloons are tested by injection of saline solution to know the minimal volume for blood flow blocking to uterine arteries. The balloons are placed distal to the superior gluteal arteries. (Reproduced with permission from Terao Y, Takeda S. Abdominal myomectomy for huge uterine myomas. In: Hiramatsu Y, Konishi I, Sakuragi N, Takeda S, eds. Mastering the Essential Surgical Procedures OGS Now, No.11. Uterine Myoma: How to Operate in These Cases? (Japanese). Tokyo: Medical View; 2012: 8-17. Copyright $\odot$ Medical View.)

Table 1 Methods to reduce bleeding during myomectomy

\begin{tabular}{|l|l|l|l|l|}
\hline \multirow{2}{*}{} & \multicolumn{2}{|l|}{$\begin{array}{l}\text { With a space in the } \\
\text { pelvic cavity }\end{array}$} & \multicolumn{2}{l|}{$\begin{array}{l}\text { Without a space in the } \\
\text { pelvic cavity }\end{array}$} \\
\cline { 2 - 5 } & Solitary & Multiple & $\begin{array}{l}\text { No elongation of the } \\
\text { cervical canal }\end{array}$ & $\begin{array}{l}\text { Elongation of the } \\
\text { cervical canal }\end{array}$ \\
\hline Vasopressin & $\bigcirc$ & $\bigcirc$ & $\bigcirc$ & $\bigcirc$ \\
\hline Clamping of the uterine artery & $\bigcirc$ & $\bigcirc$ & Difficult & Unable \\
\hline Tourniquet & $\bigcirc$ & $\bigcirc$ & Difficult & Unable \\
\hline IIABOC & Unnecessary & Unnecessary & ( & ( ) \\
\hline Autologous blood transfusion & $\triangle$ & $\bigcirc$ & $\odot$ & (-) \\
\hline
\end{tabular}

Abbreviation: IIABOC, internal iliac artery balloon occlusion catheter.

Note: @: strongly recommended; $\bigcirc$ : recommended; $\triangle$ : applicable.

Source: reproduced with permission from Takeda S. Abdominal myomectomy for huge myomas. In: Hiramatsu Y, Konishi I, Sakuragi N, Takeda S, eds. Mastering the Essential Surgical Procedures OGS Now, No.24 (Japanese). Tokyo: Medical View; 2015: 68-87. Copyright @ Medical View. 
vaginal and other arteries from external iliac artery system, that is, other than the uterine and ovarian arteries, is abundant.

\section{Tips and Warnings for Interventional Radiology}

When intra-arterial balloon occlusion in which blood flow is interrupted by placing balloon catheters in the internal or common iliac arteries, or the abdominal aorta is performed for the placenta accretes spectrum or pelvic trauma, attention should be paid to arterial thrombosis and ischemia. When balloons migrate or rupture, sufficient blood flow interruption cannot be expected in some cases. This procedure may cause artery dissection, arterial rupture, hematoma, and false aneurysm at the catheter puncture sites, as well as renal failure and allergic reactions due to the use of contrast media.

Although TAE is effective as a conservative therapy for uterine leiomyoma and hemostasis without laparotomy, it has also been reported that abortion, premature delivery, placenta accrete, uterine rupture, etc., occur in pregnancy after TAE because of poor development of the endometrium and scaring in the uterine muscle, following ischemia. ${ }^{8-10}$ At present, to our knowledge, there are no reports describing the impacts of temporary arterial balloon occlusion on subsequent pregnancies.

\section{Explanation of Procedures}

\section{Laparotomy and Exposure of the Surgical Field}

IIABOC catheters are inserted just before the operation and the balloons are deflated until myomectomy is performed. They should be firmly fixed to prevent migration. The time schedule on the day of surgery is shown in -Table 2. Because it is preferable to start the operation shortly after insertion of the occlusion catheters, close cooperation should also be established with the operating room staff.

In a patient with a cervical myoma, a huge lower corporeal myoma, or myomas with severe pelvic adhesions or complete the Douglas pouch obliteration by endometriosis, buried in the pelvic cavity, the uterus cannot be elevated even by pulling it upward. Thus, a good view of the surgical field cannot often be obtained. Furthermore, in a patient with a large myoma, there is no space between the myoma and the pelvic wall. Consequently, exposure of the retroperitoneal space may be difficult, and identification of blood vessels and ureters may not be possible. Thus, it is important to open the abdomen with a larger skin incision than the conventional size and to determine the orientation. The possible landmarks include the uterine body, Fallopian tubes, ovaries, round ligament of the uterus, and infundibulopelvic ligaments. When the orientation cannot be accurately determined, the round ligament is first severed to release the
Table 2 Example of a time schedule

\begin{tabular}{|c|c|c|}
\hline Time & \multicolumn{2}{|c|}{ Scheduled tasks } \\
\hline $\begin{array}{l}\text { Evening } \\
\text { before surgery }\end{array}$ & \multicolumn{2}{|c|}{$\begin{array}{l}\text { Insertion of an epidural catheter in } \\
\text { the operating room }\end{array}$} \\
\hline $\begin{array}{l}\text { Day of } \\
\text { surgery 09:30 h }\end{array}$ & \multicolumn{2}{|c|}{$\begin{array}{l}\text { Start insertion of balloon catheters } \\
\text { into the internal iliac arteries in the } \\
\text { angiography room at the radiology } \\
\text { department }\end{array}$} \\
\hline $10: 30 \mathrm{~h}$ & \multicolumn{2}{|c|}{$\begin{array}{l}\text { Complete catheterization. } \\
\text { Administer subcutaneous } \\
\text { injection of heparin calcium } \\
5,000 \text { units }^{\text {a }}\end{array}$} \\
\hline $10: 50 \mathrm{~h}$ & \multicolumn{2}{|c|}{$\begin{array}{l}\text { Transfer the patient to the } \\
\text { operating room. }\end{array}$} \\
\hline $11: 30 \mathrm{~h}$ & Start surgery & \multirow{2}{*}{$\begin{array}{l}\text { Duration of blood } \\
\text { flow interruption, } \\
40 \text { min }\end{array}$} \\
\hline $13: 30 \mathrm{~h}$ & $\begin{array}{l}\text { Complete } \\
\text { surgery }\end{array}$ & \\
\hline $22: 30 \mathrm{~h}$ & \multicolumn{2}{|c|}{$\begin{array}{l}\text { Administer subcutaneous injection } \\
\text { of heparin calcium } 5,000 \text { units }^{a}\end{array}$} \\
\hline $\begin{array}{l}\text { Day after } \\
\text { surgery 10:00 h }\end{array}$ & \multicolumn{2}{|c|}{$\begin{array}{l}\text { Administer subcutaneous injection } \\
\text { of heparin calcium 5,000 units, } \\
\text { start initial walks. }\end{array}$} \\
\hline
\end{tabular}

aUsed for prevention of pulmonary thromboembolism/deep vein thrombosis.

Source: reproduced with permission from Terao Y, Takeda S. Abdominal myomectomy for huge uterine myomas. In: Hiramatsu Y, Konishi I, Sakuragi N, Takeda S, eds. Mastering the Essential Surgical Procedures OGS Now, No.11. Uterine Myoma: How to Operate in These Cases? (Japanese). Tokyo: Medical View; 2012: 8-17. Copyright @ Medical View.

retroperitoneum. The infundibulopelvic ligaments, ureters, common iliac arteries, and internal iliac arteries are then identified.

\section{Tips and Warnings}

A giant myoma to which the visceral peritoneum is attached on its surface, lifts the sigmoid colon, ureters, infundibulopelvic ligaments, round ligament of the uterus, etc. On the lateral surface, the myoma is firmly attached to the dilated vascular plexus in the cardinal ligament. When the myoma grows anteriorly, it may elevate the urinary bladder. It is essential to accurately understand the relationships between the uterus and surrounding organs, based on preoperative diagnostic imaging and intraoperative macroscopic findings. If the uterus is lifted upward with excessive force, the vascular plexuses in the cardinal ligaments will be severed and cause unexpected massive bleeding.

When the precise positional relationships cannot be understood, cut-off the round ligament of the uterus to open the retroperitoneal space. This allows the infundibulopelvic ligaments and the courses of the ureters to be determined. The severed round ligament is sutured after myomectomy. 


\section{Preparation for Blood Flow Interruption}

Generally, the retroperitoneum is exposed to identify the main trunk of the uterine artery and clamp it with a blood vessel clamp. But in a patient with a giant myoma that does not allow the tourniquet method or vascular separation to be applied, such as those presented herein, occlusion catheters should be inserted preoperatively and the balloons are inflated to interrupt blood flow for at most 60 minutes. The blood flows in the ovarian arteries and veins are also interrupted temporarily.

Vasopressin (Pitressin; 0.2 units $/ \mathrm{mL}$ ) is locally injected between the capsule and the surface of a uterine leiomyoma with an $22-G$ or a $24-G$ needle. After the uterine surface discolors to white, the uterine muscle is incised.

\section{Tips and Warnings}

Measures against Massive Bleeding

Local injection of vasopressin- and adrenaline-added solution is minimally effective for reducing the volume of blood loss during myomectomy for large or multiple myomas. Instead temporarily interrupt the blood flow of the uterine arteries, as well as the ovarian arteries, as necessary. In a patient with a cervical myoma, because there is a little space between the myoma and retroperitoneum on the pelvic wall, it is impossible to expose the retroperitoneum to separate the main trunk of the uterine artery and internal iliac artery, and to tightly clamp them with blood vessel clamps. Thus, preventive measures against bleeding are difficult to implement. Furthermore, even if the cervical canal is tightly ligated at a point superior to the cervical myoma by a tourniquet, the blood flow from the descending branches of the uterine arteries cannot interrupted. This method is ineffective for preventing bleeding. Thus, to control bleeding during myomectomy and from the detachment surface, IVR procedures, such as insertion of balloon occlusion catheters, should be used.

When they make an incision up to the myoma nucleus, it is important to distinguish between the myoma and normal muscle layer and detach the myoma at the correct layer to reduce bleeding. Detachment at a muscle layer side increases the risk of bleeding.

\section{Myomectomy}

Myomectomy performed at the correct layer results in almost no bleeding. A transverse incision is made on the myoma surface up to the myoma nodule, and the myoma is detached at the border between the myoma and the membranous muscle layer. The incision is extended to obtain a space large enough to tract myoma nodule by myomectomy screw instruments or myoma borers, and the leiomyoma is held with these devices. The reason for blood vessels appearing on the surface of the myoma nodule is that the myoma is detached at a shallow layer. At a layer into the myoma nodule, detachment should be performed along the myoma. If the myoma nodule is exposed, it can easily be detached by pulling the myoma nodule and the muscle layer to be detached with myoma borers in the opposite directions (-Fig. 4).

\section{Tips and Warnings}

Practice exposing the correct layer with uterine samples obtained by total hysterectomy due to uterine leiomyomas (-Fig.4). A trick for accessing the correct layer is to make an incision until the leiomyoma nodule is incised ( - Fig. 4A). The incision is then extended to obtain a space large enough to tract myoma nodule by a myoma borer. As it is slowly moved back and forth and right and left, while being lifted upward, the border between the leiomyoma nucleus and myometrium becomes clear (-Fig. 4B). With traction on the leiomyoma maintained, it is incised with electrocautery immediately above the border. Then, the leiomyoma can be lifted further upward ( - Fig. 4C, D). The leiomyoma can be detached only by scrubbing it with gauze. After the leiomyoma has been circumferentially detached, a highly vascularized cord-like structure ultimately remains. This is held with the Pean forceps or other devices. The leiomyoma is then enucleated ( $\mathbf{- F i g . 4 E ) . ~}$

\section{Suture of the Myometrium}

To prevent hematoma formation after myomectomy, the incised muscle layers are sutured from the bottom of the space resulting from myomectomy without allowing any dead spaces to form. The first layer is sutured employing interrupted or $Z$ sutures with 0 -synthetic absorbable suture. The second and third layers may be sutured employing continuous or interlocking suture with a synthetic absorbable suture, if the dead space is wide. The dead space after enucleation, should be closed meticulously. The superficial layer is sutured by the baseball suture method (a suture technique that is based on passing a needle in the in-out pattern on the wound surface) or mattress sutures with 2-0synthetic absorbable suture. The wound surface is inverted to prevent adhesion. Depending on the depth and width of the dead space, three or five layers of uterine muscle may need to be sutured.

In the patient with a huge cervical myoma, the location of the balloon catheter for a tubal patency test should be palpated, and the muscle layer should be sutured with 2-0 or 3-0 synthetic absorbable suture in the vertical direction without suturing the cervical canal. There are some patients in whom despite the cervical mucosa having been released from the muscle layer along with the balloon catheter for a tubal patency test inserted in the cervical canal after myomectomy of a large cervical myoma, no cervical stenosis 


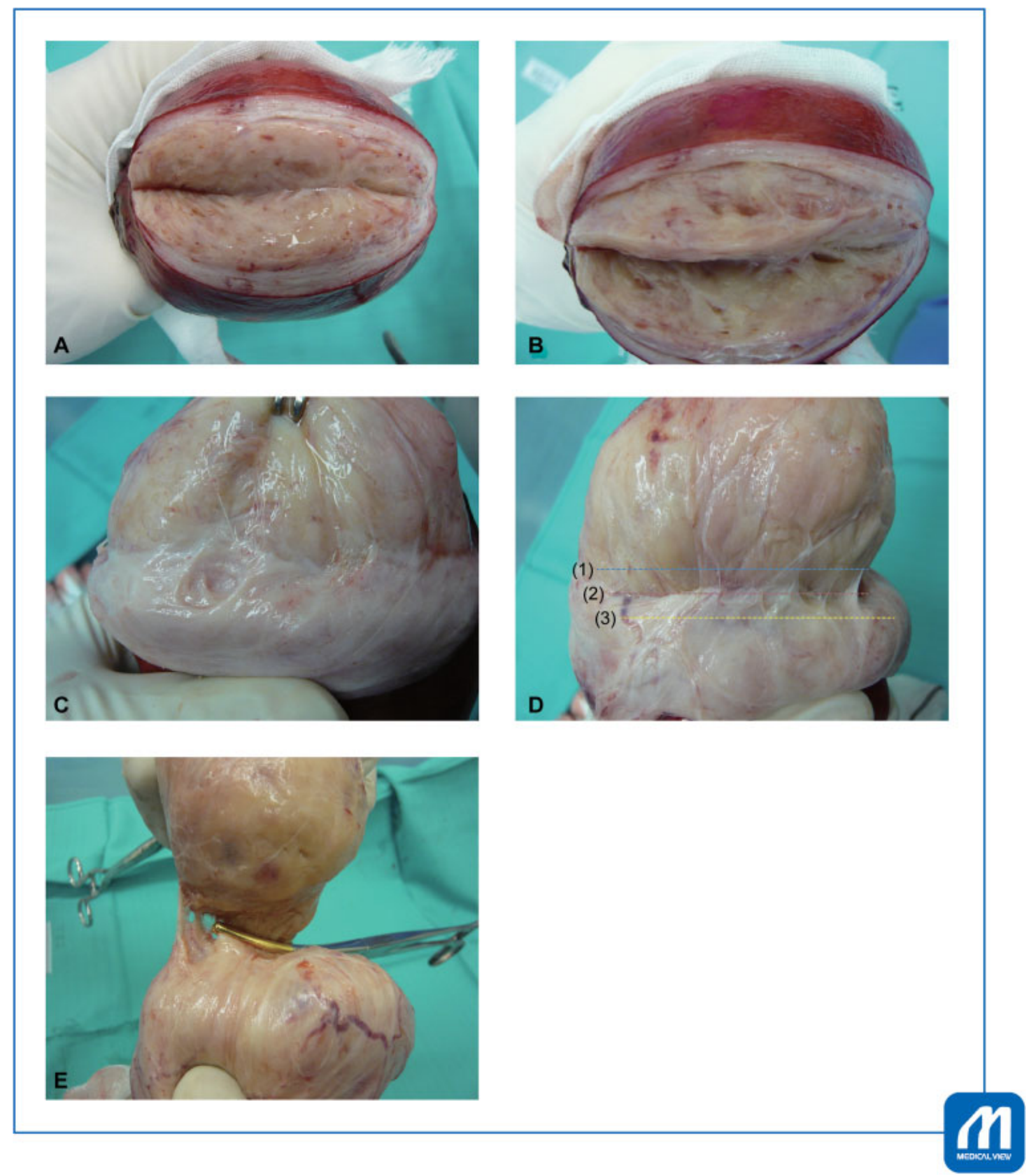

Fig. 4 Practice of myomectomy with uterine samples obtained by total hysterectomy for uterine myomas. (A) Sufficiently incise the myometrium until the inside of myoma nodule. (B) Sufficient incision facilitates holding the myoma nodule with a myoma borer. (C) If the myoma nodule is detached at the correct layer, the surface of the leiomyoma nucleus will appear shiny. (D) When the detachment process does not progress, incise line (1) with electrocautery to cut into the leiomyoma. Line (2) represents the border between the myoma and the uterine muscle, and line (3) indicates the uterine muscle. Incision around line 2 or 3 causes bleeding. (E) Finally, a highly vascularized cord-like structure remains. Hold this with Pean forceps. Enucleate the myoma nodule. Because pulling the myoma nodule too rapidly can cause bleeding, caution should be exercised. (Reproduced with permission from Terao Y, Takeda S. Abdominal myomectomy for huge uterine myomas. In: Hiramatsu Y, Konishi I, Sakuragi N, Takeda S, eds. Mastering the Essential Surgical Procedures OGS Now, No.11. Uterine Myoma: How to Operate in These Cases? (Japanese). Tokyo: Medical View; 2012: 8-17. Copyright @ Medical View.)

occurred but menstruation resumed. Thus, balloon insertion into the cervical canal is important.

Adequate meticulous closure of dead spaces after myomectomy reduces postoperative hematoma formation. After the majority of the incision has been sutured, the clamp is released and blood flow resumes, and whether there is bleeding is then examined. In a patient undergoing myomectomy for an infectious myoma or in the presence of a 


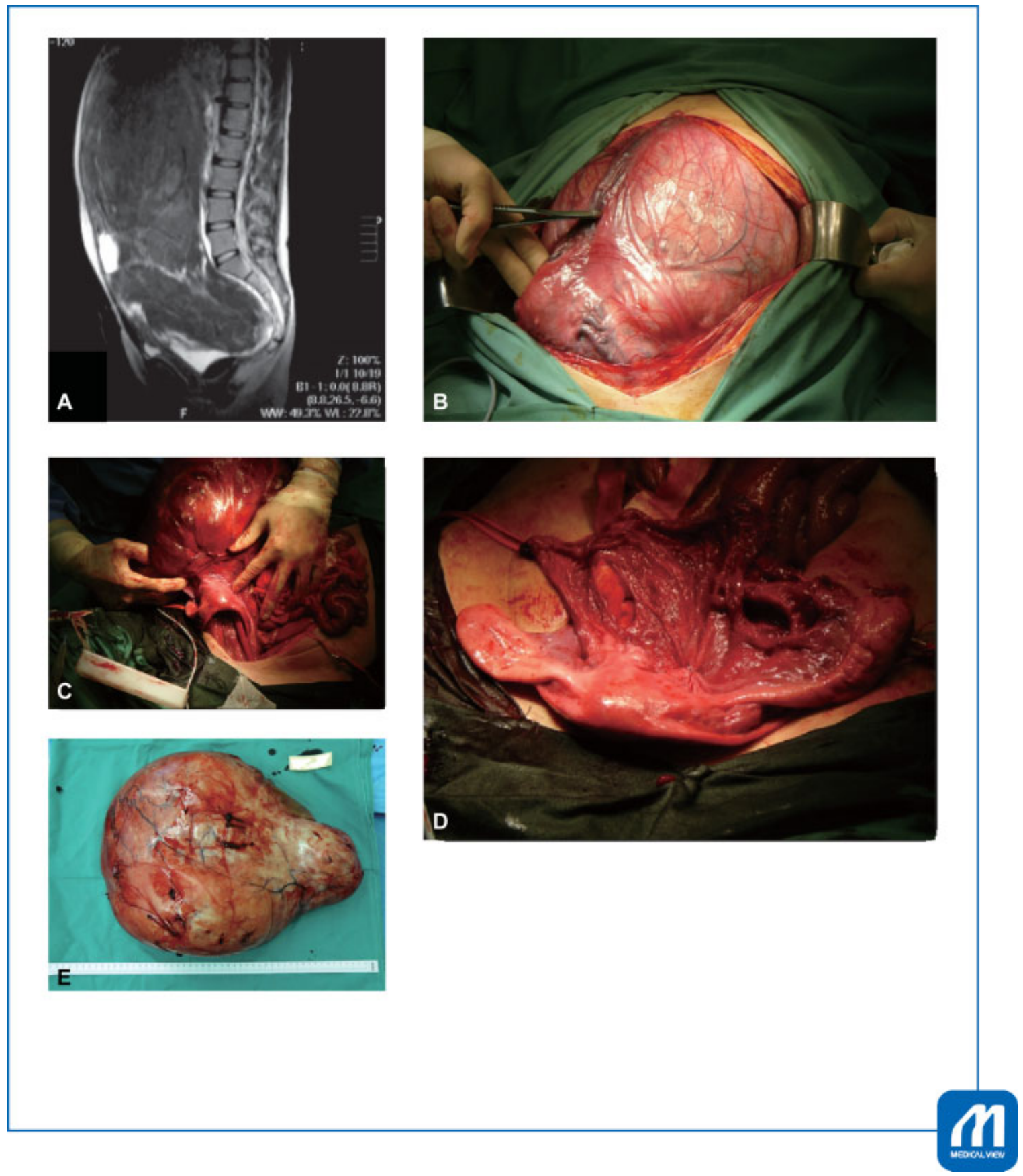

Fig. 5 Case: a huge uterine cervical myoma. (A) Findings of pelvic magnetic resonance imaging (MRI): sagittal T2-weighted MRI. A 35-cm uterine leiomyoma reaching up to the epigastric area was revealed. The cervical canal is elongated and the uterine cavity is located upward the huge cervical myoma. A huge myoma is proved to rise on the posterior wall of the cervix from the findings of pelvic MRI. (Reproduced with permission from Terao Y, Takeda S. Abdominal myomectomy for huge uterine myomas. In: Hiramatsu Y, Konishi I, Sakuragi N, Takeda S, eds. Mastering the Essential Surgical Procedures OGS Now, No.11. Uterine Myoma: How to Operate in These Cases? (Japanese). Tokyo: Medical View; 2012: 8-17. Copyright @ Medical View.) (B) Myomectomy with internal iliac artery balloon occlusion; an intraoperative view. The blood vessels on the uterine surface and around the uterus and the ovaries are dilated. (Reproduced with permission from Terao Y, Takeda S. Abdominal myomectomy for huge uterine myomas. In: Hiramatsu Y, Konishi I, Sakuragi N, Takeda S, eds. Mastering the Essential Surgical Procedures OGS Now, No.11. Uterine Myoma: How to Operate in These Cases? (Japanese). Tokyo: Medical View; 2012: 8-17. Copyright (c) Medical View.) (C) A view before myomectomy: the normal-sized ovaries and uterine corpus on the huge cervical myoma are visible. (Reproduced with permission from Takeda S. Abdominal myomectomy for huge myomas. In: Hiramatsu Y, Konishi I, Sakuragi N, Takeda S, eds. Mastering the Essential Surgical Procedures OGS Now, No.24. (Japanese). Tokyo: Medical View; 2015: 68-87. Copyright @ Medical View. (D) A view after myomectomy: after myomectomy, the balloon catheter for a tubal patency test was inserted into the uterine cavity, and the muscle layer was sutured with synthetic absorbable sutures in the vertical direction without suturing the cervical canal. (Reproduced with permission from Takeda S. Abdominal myomectomy for huge myomas. In: Hiramatsu Y, Konishi I, Sakuragi N, Takeda S, eds. Mastering the Essential Surgical Procedures OGS Now, No.24 (Japanese). Tokyo: Medical View; 2015: 68-87. Copyright (C) Medical View). (E) A picture of the resected specimen. The leiomyoma weighed $10.5 \mathrm{~kg}$. (Reproduced with permission from Takeda S. Abdominal myomectomy for huge myomas. In: Hiramatsu Y, Konishi I, Sakuragi N, Takeda S, eds. Mastering the Essential Surgical Procedures OGS Now, No.24 (Japanese). Tokyo: Medical View; 2015: 68-87. Copyright @ Medical View). 
large residual dead space, a continuous closed suction drain may be inserted and left in place for 1 to 2 days in the muscle layer that is the site of myomectomy.

\section{Tips and Warnings}

Myomectomy and subsequent pregnancy:

The midline portion of the uterus is the most distal portion with regards to blood supply from the right and left uterine arteries. This portion is a common site of uterine scar rupture in pregnancy after Cesarean section, and uterine ischemia and uterine rupture due to TAE also predominantly occurs in the midline portion. For patients contemplating a subsequent pregnancy, we think that a myometrial incision should, in principle, be made employing a transverse incision and closed with a vertical suture because of scar formation. The uterine fundus is also a watershed with regards to the distribution of blood flow from the right and left uterine arteries. It can be regarded as distal to the blood supply.

High incidences of placenta accreta spectrum, such as placenta previa increta, placenta percreta, and uterine rupture have been reported after myomectomy of a myoma in the muscle layer of the uterine fundus. Although these observations need to be confirmed by large-scale surveys, the above conditions should be kept in mind as potential complications of pregnancy after myomectomy. In the uterine fundus, an incision should be made employing a transverse incision and closed with a vertical suture.

When managing a postoperative pregnancy, following cervical myomectomy, pay attention to the possibility of cervical incompetency. The muscle layer of the uterine cervix is weak, and vaginal delivery might be associated with the risk of the cervical laceration and uterine rupture. Thus, cesarean section should be undertaken.

\section{Closure of the Abdomen}

The peritoneal cavity is sufficiently irrigated with 2,000 to $3,000 \mathrm{~mL}$ of physiological saline. If bleeding is detected, ligation and suturing are performed to ensure hemostasis.

A continuous closed suction drain is inserted into the Douglas pouch. If no bleeding is observed, the drain will be removed the following day.

Absorbable adhesion barrier, such as Interceed or Seprafilm is applied to the wounds on the uterus and the abdominal wall, and the abdomen is closed. The rates of preventing adhesion differ minimally among these materials. The uterus is covered with the greater omentum, and the abdomen is closed.

\section{Case: A Huge Uterine Cervical Myoma}

A 31-year-old woman, gravida 0 and para 0 , had noted the presence of an abdominal mass for 1 year but it had been left untreated. She visited our hospital with severe bloating. An abdominal mass reaching up to the xiphoid process was confirmed. MRI revealed a $35-\mathrm{cm}$ uterine leiomyoma (-Fig. 5A). She strongly desired myomectomy. After GnRH agonist therapy had been administered five times, balloon catheters were bilaterally placed in the internal iliac arteries before surgery. Both balloons were inflated immediately before myomectomy ( $\mathbf{F i g . 5 B}, \mathbf{C}$ ). Then, a transverse incision was performed on the surface of the cervical myoma nodule and myomectomy was performed (-Fig. 5D). After myomectomy, the balloon catheter for a tubal patency test was inserted into the uterine cavity, and the muscle layer was sutured with 2-0 or 3-0 synthetic absorbable suture in the vertical direction without suturing the cervical canal. The operation time was 2 hours 43 minutes and the blood flow blocking time by IIABO was 35 minutes. The resected leiomyoma weighed 10,500 g (-Fig. 5E), and the blood loss volume was $505 \mathrm{~g}$. Although she was discharged on postoperative day 8 , she developed ileus symptoms and was readmitted on postoperative day 14 . Her condition improved with 3 days of fasting, and then the postoperative course was uneventful.

\section{Conclusion}

Although uterine leiomyomas are benign, the difficulty in performing myomectomy varies depending on the size and site of a leiomyoma. In some cases, it is very challenging. While sufficiently performing preoperative assessment with pelvic examination, ultrasonography, MRI, etc., surgeons should always keep in mind how they can reduce the blood loss volume, while safely and surely performing resections. For a cervical myoma of the uterus and giant uterine leiomyoma that leave no intrapelvic space and prevent palpation and identification of the uterine arteries and the internal iliac arteries, surgery can be performed safely by preoperatively placing balloon catheters in the internal iliac arteries. $^{1,3}$ Thus, if this procedure is regarded as one of the feasible approaches, the procedural options for performing myomectomy will likely further increase.

Conflict of Interest

None.

\section{References}

1 Ota T, Kaneda H. Temporary endovascular balloon occlusion of the internal iliac arteries to prevent hemorrhage during uterine cervical myomectomy. In: Takeda S, Kuwatsuru R, eds. Gynecologic and Obstetric Prophylactic Hemostasis by Intra-arterial Balloon Occlusion. 1st ed. Singapore: Springer; 2018:23-31

2 Kyogoku S. Application and methods of arterial embolization for prophylaxis and treatment of massive hemorrhage in obstetric field. In: Takeda S, Kuwatsuru R eds. Gynecologic and Obstetric Prophylactic Hemostasis by Intra-arterial Balloon Occlusion. 1st ed. Singapore: Springer; 2018:9-21

3 Kaneda H, Terao Y, Matsuda Y, et al. The utility and effectiveness of an internal iliac artery balloon occlusion catheter in surgery for large cervical uterine fibroids. Taiwan J Obstet Gynecol 2017;56 (04):502-507 
4 Takeda J, Makino S. Temporary arterial balloon occlusion for obstetrical field. In: Takeda S, Kuwatsuru R, eds. Gynecologic and Obstetric Prophylactic Hemostasis by Intra-arterial Balloon Occlusion. 1st ed. Singapore: Springer; 2018:33-39

5 Shrivastava VK, Nageotte MP. The utilization of interventional radiologic procedures in the surgical management of placenta accreta syndrome. Clin Obstet Gynecol 2018;61(04):795-807

6 Sone M, Nakajima Y, Woodhams R, et al. Interventional radiology for critical hemorrhage in obstetrics: Japanese Society of Interventional Radiology (JSIR) procedural guidelines. Jpn J Radiol 2015;33(04):233-240

7 Salazar GM, Petrozza C, Walker TG. Transcatheter endovascular techniques $\mathrm{f}$ o $\mathrm{r}$ management of obstetrical and gynecologic emergencies. Tech Vasc Interv Radiol 2009;12(02):139-147
8 Inoue S, Masuyama H, Hiramatsu Y; Multi-Institutional Study Group of Transarterial Embolization for Massive Obstetric Haemorrhage in Chugoku \& Shikoku Area Society of Obstetrics and Gynecology. Efficacy of transarterial embolisation in the management of post-partum haemorrhage and its impact on subsequent pregnancies. Aust N Z J Obstet Gynaecol 2014;54(06):541-545

9 Takeda J, Makino S, Ota A, Tawada T, Mitsuhashi N, Takeda S. Spontaneous uterine rupture at 32 weeks of gestation after previous uterine artery embolization. J Obstet Gynaecol Res 2014;40(01):243-246

10 Sano Y, Takeda J, Kuroda K, Makino S, Itakura A, Takeda S. Embrittlement of uterus after uterine artery embolization: a case of uterine perforation. Hypertens Res Pregnancy 2016;4 (01):42-44 